\title{
Overcoming Interaction Blindness through Curiosity Objects
}
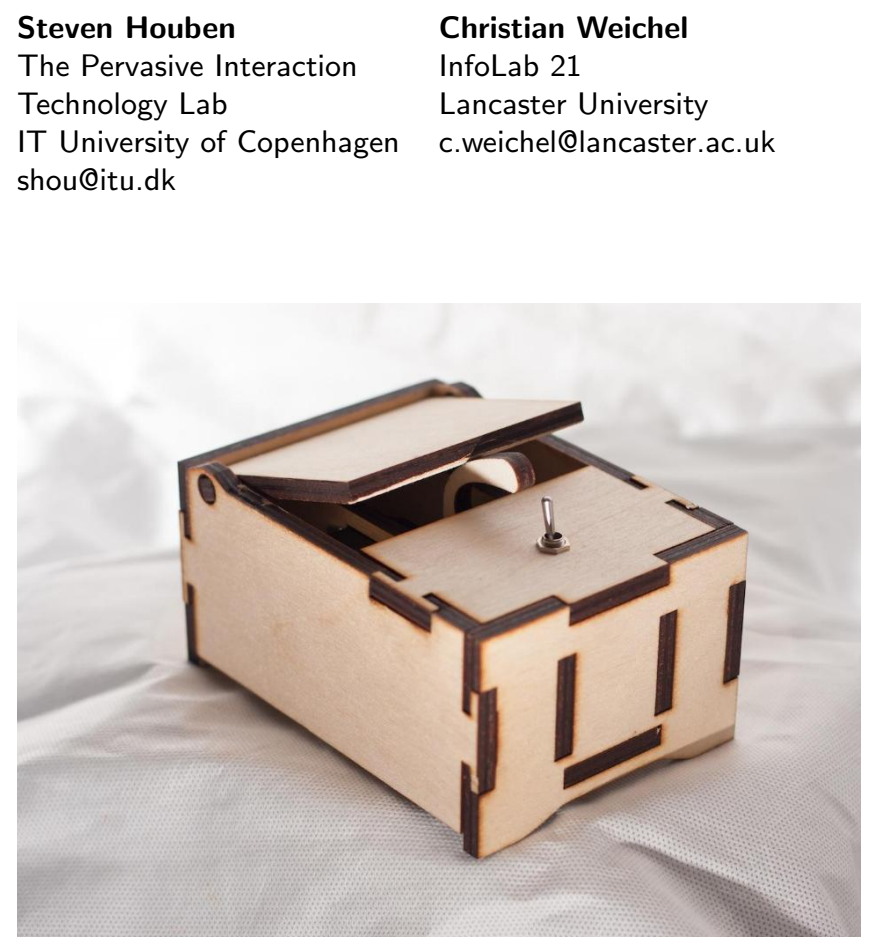

Copyright is held by the author/owner(s).

CHI'13, April 27 - May 2, 2013, Paris, France.

ACM 978-1-4503-1952-2/13/04.

\begin{abstract}
In recent years there has been a widespread installation of large interactive public displays. Longitudinal studies however show that these interactive displays suffer from interaction blindness - the inability of the public to recognize the interactive capabilities of those surfaces. In this paper, we explore the use of curiosity-provoking artifacts, (curiosity objects) to overcome interaction blindness. Our study confirmed the interaction blindness problem and shows that introducing a curiosity object results in a significant increase in interactivity with the display as well as changes in movement in the spaces surrounding the interactive display.
\end{abstract}

\section{Author Keywords}

display blindness, interaction blindness, curiosity object, situated public displays

\section{ACM Classification Keywords}

H.5.2 [Information interfaces and presentation]: Input devices and strategies; H.1.2 [User/Machine Systems]: Human factors

\section{Introduction}

In the last decade, public displays have increasingly been introduced in urban spaces around the world. These screens are typically used to display information (e.g. in 


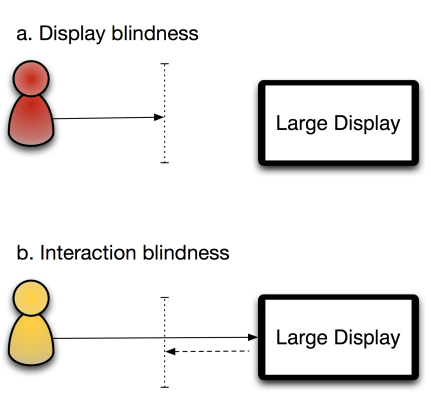

Figure 1: A conceptualization of the display/interaction blindness problem. train stations or airports), advertisement (e.g. in shopping malls) or video and television [2]. With the introduction of touch technology, the traditionally unidirectional communication of these public displays have been altered to a two-way communication that allows public interaction.

Despite the ubiquity of these interactive displays, longitudinal studies $[6,11]$ have shown that these types of urban displays suffer from substantial problems. First, because the majority of public displays are used for advertisement and publicity purposes, they elicit display blindness [9] (Figure 1 a): many people tend to only quickly glance or even ignore these display as the contained information is perceived as unimportant or irrelevant [6]. Second, interaction blindness [11] (Figure 1 b), a related problem, is caused by the fact that interactive displays often look like non-interactive displays, resulting in users to be unaware of a systems interactivity. This problem is potentially even amplified in cases were public displays switch between publicity and interactive mode.

In literature, several approaches for overcoming display and interaction blindness have been described. Explicit interaction invitations, e.g. a "touch me" message on the screen, suffer from two major drawbacks: for one, they require screen estate which is not feasible in many advertisement scenarios. Second, displaying invitations alone are ineffective [11]. Another approach is to integrate portable devices, such as phones, into the interaction [5]. They do however, require active interruption of the user to overcome the blindness problem. Other approaches include context-aware systems using location tracking, gaze activation [8] or movement [7].

In general, there is a mismatch between the general public's perception and the actual functionality of interactive displays. This mismatch influences the level of exploration and curiosity people demonstrate for these screens. We propose the notion of a curiosity object, a curiosity invoking object that is designed to passively attract people and remove the display/interaction blindness of interactive displays.

In this paper we give further evidence for the problem of display and interaction blindness, introduce the notion of curiosity objects and explore how curiosity objects can decrease the display/interaction blindness problem.

\section{Curiosity as Motivator}

Design for Curiosity

Curiosity is one of the important driving factors of human behaviour as it is used as mechanism to make sense of the world [1]. It is stimulated by external conflicting stimuli such as complexity, novelty, and surprise and influences how people interact with physical objects. Summarized, perceptual curiosity is the attention and interest given to a novel perceptual stimulation that motivates sensory and visual inspection.

Based on this theoretical work of Berlyne [1], Tieben et al. [12] propose five properties: (i) novelty, (ii) complexity, (iii) uncertainty, (iv) conflict and (v) partial exposure, as fundamental principles to design for curiosity. Their description of the curiosity process is composed of different phases that are directly influenced by these principles. At first, humans encounter a curious situation driven by the novelty, uncertainty and conflict of that particular situation. After this initialization phase, they explore and discover the situation influenced by the complexity and exposure. The latter two thus determine the lasting effect of the exploration that resulted from the curiosity. 
The importance of curiosity as an intrinsic motivation for interactive technology has also been recognized by Muller et al. [8]. Their design space analysis reveal that curiosity "belongs to the most important characteristics of intrinsically motivating environments" and describe how well crafted interaction can induce curiosity and motivate people to engage into interaction with large displays.

Inspired and motivated by this previous work, we propose the notion of a curiosity object, an object, informed by principles of Tieben et al. [12], that is used as a mediator between the public and interactive displays in an effort to remove the display and interaction blindness. Because of its curious character, it has a honey pot effect as it attracts people based on its natural properties and affordances. When people interact with the device, the curiosity object reveals the interactive possibilities of the displays, thereby removing the display and interaction blindness.

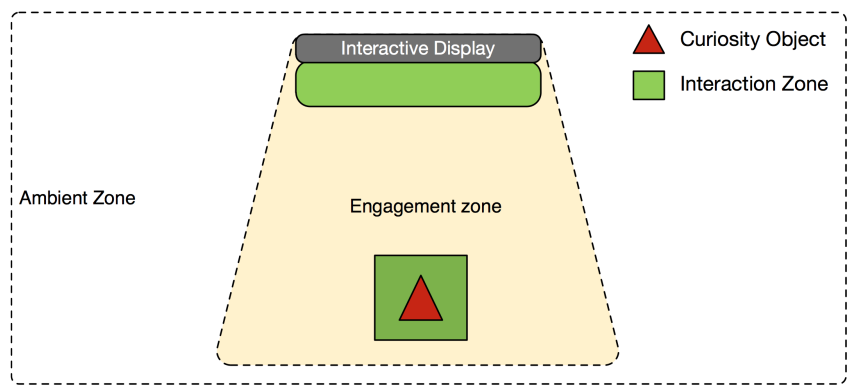

Figure 2: The positioning of the display and curiosity object creates four zones.

Zones

The positioning of both the public interactive display and curiosity object creates a number of zones (Figure 2)
$[4,10]$. The primary interaction zone is directly in front of the interactive display allowing a person to physically

touch the screen. The secondary interaction zone refers to the space surrounding the curiosity object. People in this space are able to touch and interact with the object. The engagement zone is the surrounding space in which people can observe the content of the display. Finally, the ambient zone refers to the physical space in which people are able to observe the displays presence but not its content.

\section{Experimental Setup}

To explore the effects of a curiosity object on the visibility of the interactive possibilities of an interactive display, we conducted a two-day experiment. The purpose of this exploratory experiment is twofold: (i) create a baseline that provides further evidence for the existence of the display/interaction blindness problem and (ii) explore the short-term effects of a curiosity object compared to this baseline.

In this paper we reconceptualize an artifact known as the "Worlds Most Useless machine" (WMU machine) to a curiosity object (front page figure). The machine was invented by Claude Shannon and initially described by Arthur C. Clarke [3]. The machine is a small wooden casket, the size of cigar box, that only contains a switch at one side of the top plane, and a servo actuated arm, that remains hidden inside the device enclosure. Once a user toggles the switch, the machine actuates its arm, pushing the lid open, to restore the state of the switch to the off-position, effectively undoing the users action and rendering the machine useless. The "Worlds Most Useless machine" fulfils all five curiosity qualities. Despite its increasing popularity, it is still fairly novel. The switch-reset mechanism exhibits a certain degree of complexity, but not so much that the machine would be 

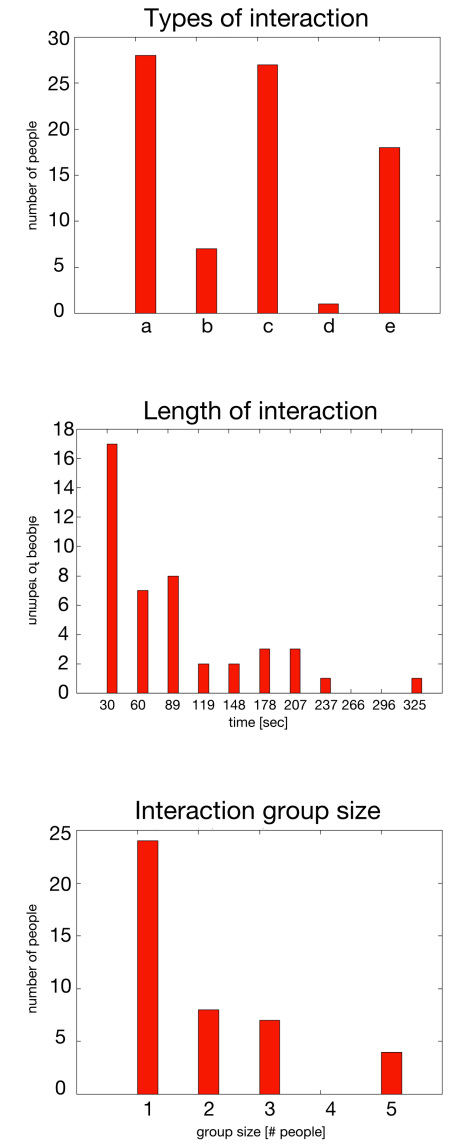

Figure 4: The distribution of interaction types (labelled according the scenarios in table 1 ), interaction durations and amount of people involved in each interaction instance puzzling. Its shape and the switch it contains result in uncertainty and conflict as the result of actuating the switch is not clear beforehand.

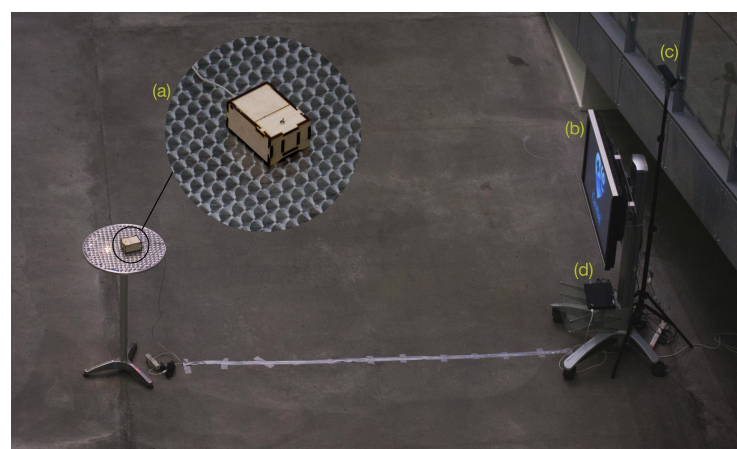

Figure 3: The experimental setup in the curiosity variant. (a) the curiosity object, (b) the interactive display in poster mode (c) the Microsoft Kinect sensor placed so it can detect users even when they're interacting with the screen and (d) a laptop connected to the display running the software.

Our system (Figure 3) has two variants. First, the baseline-variant consists of a display and a Microsoft Kinect depth-sensor used to gather movement data. The second variant is the curiosity variant which extends the baseline-version with a curiosity object (in this case the "worlds most useless machine"). In both setups, the Microsoft Kinect is used to reset the experiment when there is no user within a 3 meter range. Resetting the experiment causes the WMU machine to reset the switch state if necessary. This simple baseline setting is based on observations on the setup of the public displays that are deployed in Oulu [11].

In both variants the interactive touch screen runs in two modes: (i) poster mode, in which it would show advertisement-like information and (ii) interactive mode, in which users can sketch using touch interaction. In the baseline the system switches from poster to interactive mode by touching the screen. Whereas in the curiosity variant, the display goes into interactive mode when a user actuates the WMU machine switch or touches the screen. If a user toggles the WMU machine switch but does not touch the screen, the screen will go to poster mode after one minute. In order to deploy the curiosity object, we added a table and connection between the curiosity object and display. We used a table that could be found in the atrium and covered the cable connecting the curiosity object and display in the same color as the floor.

We deployed the system on two consecutive days in the atrium of the IT University of Copenhagen. On the first day, we installed the baseline version at 7:50 in the morning to avoid drawing unnecessary attention on the newly introduced system. The experiment was left running until 16:30 under constant observation with a video camera placed three stories above ground floor so that we would remain undetected. On the second day, we deployed the curiosity variant as shown in figure 3 - again at 07:50. We ran the experiment until 16:00 while occupying the same observation post as the day before.

The experiment was recorded using a video camera and the captured video was annotated manually. Additionally, the system was setup to log data from the Kinect skeleton tracking, WMU machine interaction and screen interaction.

\section{Results}

During the two day deployment, we observed approximately 1600 participants (861 passer-bys on day 1 and 825 on day 2). During the baseline variant (day 1 ), not a single person interacted with the display whereas during the curiosity object variant (day 2) 41 interaction 

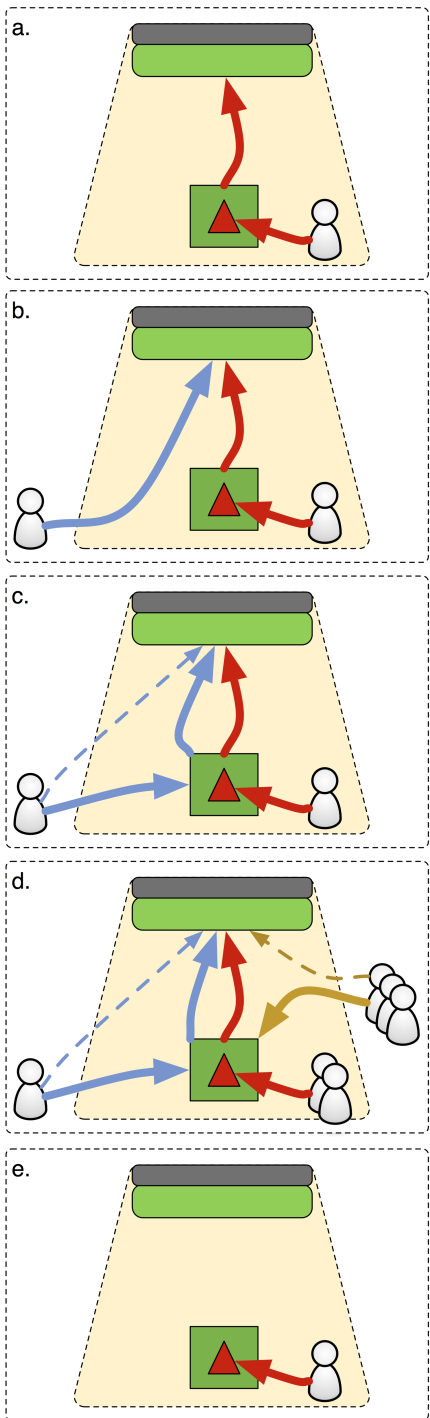

Figure 5: Different zone movements caused by the curiosity object (named as shown in table 1). instances (activation of interactive mode was logged by the system) involving 81 people occurred. Table 1 and Figure 4 provides a overview of the types of interactions that were observed over the course of one day.

\begin{tabular}{ll}
\hline Interaction scenario & $\begin{array}{l}\text { Instance } \\
\text { count }\end{array}$ \\
\hline (a) primary (display) interaction & 28 \\
(b) primary interaction with social effects & 7 \\
(c) primary and secondary (curiosity object) & 27 \\
interaction with social effects & \\
(d) primary and secondary interaction with & 1 \\
group dynamics & 18 \\
(e) secondary interaction only & 18 \\
\hline
\end{tabular}

Table 1: An overview of the five different interaction scenarios we observed during the second day of the experiment.

\section{Discussion}

During the deployment of the base variant, we were able to confirm both the display [6] and interaction [11] blindness problems. Almost all passers-by completely ignored the display and the few people that did notice the screen, did not realize it was interactive or were reluctant to walk up to the display and interact with it. During the baseline deployment no-one interacted with the display.

There was an increase in interactivity in the system variant that contained the curiosity object. Many participants were attracted to the curiosity object and tried to interact with it. Because of this initial interaction, the interactive possibilities of the display were highlighted, causing people to move up to the screen and make a drawing. In total 81 people interacted individually or in a group with the setup resulting in 78 sketches (Figure 6). Analysis of the video recordings in relation to the aforementioned zones showed
5 distinct patterns (Figure 5) in which people interacted with both the curiosity object and the interactive display.

$76 \%$ of the participants that were attracted by the curiosity object (secondary interaction) also moved to the screen to create a sketch (primary interaction). This movement from the curiosity object to the primary screen is one of the main observations that confirms the ability of the curiosity object to (partially) remove the display and interaction blindness. However, in $24 \%$ of the cases, participants would interact only with the curiosity object, ignoring the interactive display. These were primarily passers-by that simply flipped the switch without waiting for a response or people who simply did not find the screen interesting enough.

A side effect of people interacting with the main display (after using the curiosity object) is that some passers-by noticed the emerging or ongoing interaction, and are directly attracted by the display without even noticing the curiosity object. This shows that the curiosity level of the object is balanced enough to start the exposure of the interactivity of the display but not to suck up the attention of the main interaction actors and passers-by. The primary interaction thus produces social effects that draws other people to the display.

The interactive display and curiosity object is devised to reset after interaction actors walk away from the screen. The curiosity object shows a visual cue of reset (in this case, the automatic reset of the toggle switch) which can be noticed by passers-by. We observed several instances where the visual reset of the curiosity object would draw attention of people who would then again start interaction with the setup as mentioned in the first two patterns. During one instance, the social effect described in pattern $b$ and $c$ (Figure 5) snowballed into a large group that 


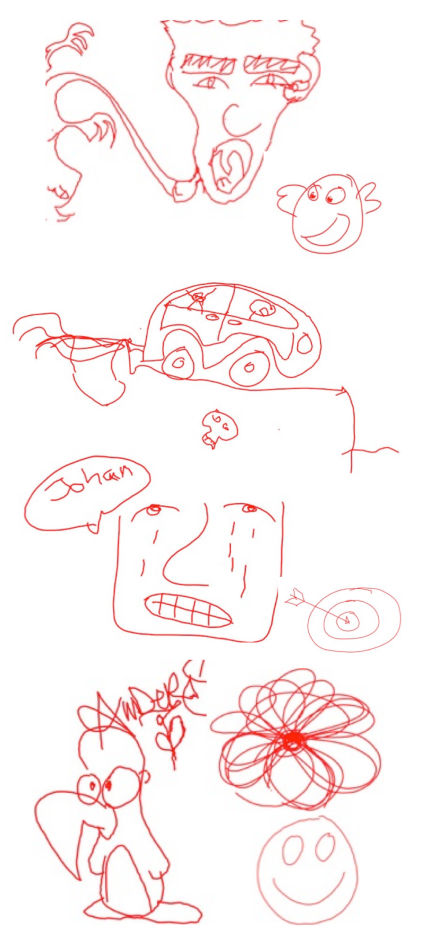

Figure 6: A sample of the sketches made once the interactive abilities were exposed would form around the curiosity object and the interactive screen (Figure $5 \mathrm{e}$ ) . During this instance, the social effect was amplified because of the presence of a crowd.

Finally, towards the end of the experiment, we noticed how passers-by would simply ignore the curiosity object and walk up to the display to create a sketch. It seemed that even in this one day deployment the curiosity object succeeded in revealing the interactive capabilities of the screen to some people.

\section{Future Work}

Previous work has demonstrated the potential and value of curiosity as an intrinsic motivator for people to interact with digital systems. In this paper we explored the notion of a curiosity object as a mediating artifact between the public and large public interactive displays. Our exploratory study showed that the display and interaction blindness problem can be reduced by attaching a curiosity object to the interactive display. However, a number of open questions emerged from this work regarding the form factor, phases of curiosity, deployment methods, socia effects and long-term effectiveness. Future work thus includes reproducing the study results in different locations with different types of curiosity objects and performing longitudinal studies to explore the long term effect of curiosity objects.

\section{Acknowledgements}

This work was supported by the EU Marie Curie Network iCareNet under grant number 264738.

\section{References}

[1] Berlyne, D. Conflict, arousal, and curiosity. McGraw-Hill, 1960.

[2] Brignull, H., and Rogers, Y. Enticing people to interact with large public displays in public spaces. In In proc. of Interact '03, IOS Press (2003), 17-24.
[3] Clark, C. A. Voice Across the Sea. William Luscombe Publisher Ltd, 1974

[4] Hincapié Ramos, J. D., Tabard, A., and Bardram, J. E. Gridorbit: an infrastructure awareness system for increasing contribution in volunteer computing. In In proc. of CHI, ACM (New York, NY, USA, 2011), 1899-1908.

[5] Holleis, P., Rukzio, E., Otto, F., and Schmidt, A. Privacy and curiosity in mobile interactions with public displays. In In proc. of $\mathrm{CHI}$ '07 workshop on Mobile Spatial Interaction (2007)

[6] Huang, E. M., Koster, A., and Borchers, J. Overcoming assumptions and uncovering practices: When does the public really look at public displays? In In proc. of PerCom '08, Springer-Verlag (Berlin, Heidelberg, 2008), 228-243.

[7] Ju, W., and Sirkin, D. Animate objects: how physical motion encourages public interaction. In proc of Persuasive Technology '10, Springer-Verlag (Berlin, Heidelberg, 2010), 40-51.

[8] Müller, J., Alt, F., Michelis, D., and Schmidt, A. Requirements and design space for interactive public displays. In In proc. of MM '10, ACM (New York, NY USA, 2010), 1285-1294.

[9] Müller, J., Wilmsmann, D., Exeler, J., Buzeck, M., Schmidt, A., Jay, T., and Krüger, A. Display blindness: The effect of expectations on attention towards digital signage. In In proc. of PerCom '09, Springer-Verlag (Berlin, Heidelberg, 2009), 1-8.

[10] O'Hara, K., Perry, M., Churchill, E., and Russell, D. Public and Situated Displays: Social and Interactional Aspects of Shared Display Technologies. Springer Publishing Company, Incorporated, 2011.

[11] Ojala, T., and Kostakos, V. e. a. Multipurpose interactive public displays in the wild: Three years later. Computer (2012).

[12] Tieben, R., Bekker, T., and Schouten, B. Curiosity and interaction: making people curious through interactive systems. In proc. of $\mathrm{BCS} \mathrm{HCl}$ '11, British Computer Society (2011). 